\title{
High Frequency Assessment from Multiresolution Analysis
}

Tássio Knop de Castro, Eder de Almeida Perez, Virgínia Fernandes Mota, Alexandre Chapiro, Marcelo Bernardes Vieira, and Wilhelm Passarella Freire

Universidade Federal de Juiz de Fora, DCC/ICE, Cidade Universitária, CEP: 36036-330, Juiz de Fora, MG, Brazil

\{tassioknop, eder . perez, virginia.fernandes, alexandre.chapiro, marcelo.bernardes, wilhelm.freire\}@ice.ufjf.br

http://www.gcg.ufjf.br

\begin{abstract}
We propose a method for the assessment and visualization of high frequency regions of a multiresolution image. We combine both orientation tensor and multiresolution analysis to give a scalar descriptor of high frequency regions. High values of this scalar space indicate regions having coincident detail vectors in multiple scales of a wavelet decomposition. This is useful for finding edges, textures, collinear structures and salient regions for computer vision methods. The image is decomposed into several scales using the Discrete Wavelet Transform (DWT). The resulting detail spaces form vectors indicating intensity variations which are combined using orientation tensors. A high frequency scalar descriptor is then obtained from the resulting tensor for each original image pixel. Our results show that this descriptor indicates areas having relevant intensity variation in multiple scales.
\end{abstract}

Keywords: high frequency detection, multiresolution analysis, orientation tensor.

\section{Introduction}

The evaluation of high frequencies in an image is an important task for several applications in computer vision, computer graphics and image processing. Objects in a scene are mainly distinguished by the contrast of their borders against a background. In a signal processing point of view, this can be seen as brightness variation with multiple frequencies.

However, object and background areas can be arbitrarily complex. One way of estimating salient regions is to use multiresolution to capture global and local brightness variations. Even in a non-redundant wavelet decomposition, local and global borders occurring in the same region may carry useful information. The problem is to combine the global information into a single image. In this sense, orientation tensors can capture the multivariate information of several scales and color channels [1].

In this paper, we combine both orientation tensor and multiresolution analysis to give a scalar descriptor of high frequency regions. High values of this

G. Allen et al. (Eds.): ICCS 2009, Part I, LNCS 5544, pp. 429 438, 2009.

(C) Springer-Verlag Berlin Heidelberg 2009 
scalar space indicate regions having coincident detail vectors in multiple scales of wavelet decomposition. This is useful for finding edges, textures, collinear structures and salient regions for computer vision methods.

\section{Related Work}

Orientation tensors can be used to analyze and draw conclusions about the quality of an image. In Fronthaler et al. [2, the objective is to distinguish noisy content from possible non-trivial structures in biometric assessments. The orientation tensor is decomposed into symmetric representations from which a particular definition of quality can be estimated.

Wong and Chung [3] use orientation tensors to exploit local structural coherence to improve the quality of the binary segmentation of an image. An estimation of the local structural orientation through eigen decomposition of these tensors is performed for local structure description.

In Han and Shi [4], the wavelet transform plays an important role in the task of decomposing a texture image into several levels. Once a decomposition level is chosen, textures are then removed from the original image by the reconstruction of low frequencies only.

Bigun et al. 5] use a structure tensor to represent and detect more intricate patterns than straight lines and edges to produce and filter dense orientation fields for feature extraction, matching, and pattern recognition.

Schou et al. 6] propose a method to detect line and edge structures in multichannel remote sensing images. They also use tensors to represent orientation information. Vliet and Faas [7] decompose structure tensors to analyze and represent multiple oriented structures inside a local neighborhood of an image. They propose cluster analysis to divide the local gradient vectors that would normally construct a single tensor into a limited number of clusters.

Most of the related works use orientation tensors or multiresolution as a step to gather specific image information in a single scale. In this paper, a weighted sum of orientation tensors, obtained from several multiresolution scales, is used to combine high frequencies in only one tensor field. This resulting tensor field captures regions having coincident high frequencies that can be used to detect salient areas.

\section{Fundamentals}

\subsection{Wavelets}

The wavelet transform decomposes signals over dilated and translated wavelets [8]. A wavelet is a function $\psi \in L^{2}(\Re)$ with a zero average:

$$
\int_{-\infty}^{+\infty} \psi(t) d t=0
$$


It is normalized $\|\psi\|=1$, and centered in the neighborhood of $t=0$. A family of time-frequency atoms is obtained by scaling $\psi$ by $s$ and translating it by $u$ :

$$
\psi_{u, s}(t)=\frac{1}{\sqrt{s}} \psi\left(\frac{t-u}{s}\right)
$$

We are interested in wavelets which form a base of $L^{2}\left(\Re^{2}\right)$ to represent images. If we have an orthonormal wavelet basis in $L^{2}(\Re)$ given by $\psi$ with the scaling function $\phi$, we can use

$$
\psi^{1}(x)=\phi\left(x_{1}\right) \psi\left(x_{2}\right), \psi^{2}(x)=\psi\left(x_{1}\right) \phi\left(x_{2}\right), \psi^{3}(x)=\psi\left(x_{1}\right) \psi\left(x_{2}\right)
$$

to form an orthonormal basis in $L^{2}\left(\Re^{2}\right)$ [8].

$$
\left\{\psi_{j, p}^{1}, \psi_{j, p}^{2}, \psi_{j, p}^{3}\right\}_{[j, p] \in Z^{3}}
$$

In this paper, we define a vector $v_{j, p} \in \Re^{3}$ given by the inner product

$$
v_{j, p}=\left[I \cdot \psi_{j, p}^{1}, I \cdot \psi_{j, p}^{2}, I \cdot \psi_{j, p}^{3}\right]^{T}
$$

at scale $j$ and position $p \in I$, where $I$ is the input image.

\subsection{Orientation Tensor}

A local orientation tensor is a special case of non-negative symmetric rank 2 tensor, built based on information gathered from an image. As shown by Knutsson [1, such a tensor can be produced by combining outputs from polar separable quadrature filters. Because of its construction, such a tensor has special properties and contains valuable information about said image.

From the definition given by Westin [9], orientation tensors are symmetric, and thus an orientation tensor $T$ can be decomposed using the Spectral Theorem as shown in (6), where $\lambda_{i}$ are the eigenvalues of $T$.

$$
T=\sum_{i=1}^{n} \lambda_{i} T_{i}
$$

If $T_{i}$ projects onto a $m$-dimensional eigenspace, we may decompose it as

$$
T_{i}=\sum_{s=1}^{m} e_{s} e_{s}^{T}
$$

where $\left\{e_{1}, \ldots, e_{m}\right\}$ is a base of $\Re^{m}$. An interesting decomposition of the orientation tensor $T$ proposed by Westin [9] is given by

$$
T=\lambda_{n} T_{n}+\sum_{i=1}^{n-1}\left(\lambda_{i}-\lambda_{i+1}\right) T_{i}
$$

where $\lambda_{i}$ are the eigenvalues corresponding to each eigenvector $e_{i}$. This is an interesting decomposition because of its geometric interpretation. In fact, in $\Re^{3}$, 
an orientation tensor $T$ decomposed using (8) can be represented by a spear (its main orientation), a plate and a ball

$$
T=\left(\lambda_{1}-\lambda_{2}\right) T_{1}+\left(\lambda_{2}-\lambda_{3}\right) T_{2}+\lambda_{3} T_{3} .
$$

A $\Re^{3}$ tensor decomposed by (9), with eigenvalues $\lambda_{1} \geq \lambda_{2} \geq \lambda_{3}$, can be interpreted as following:

$-\lambda_{1}>>\lambda_{2} \approx \lambda_{3}$ corresponds to an approximately linear tensor, with the spear component being dominant.

$-\lambda_{1} \approx \lambda_{2}>>\lambda_{3}$ corresponds to an approximately planar tensor, with the plate component being dominant.

$-\lambda_{1} \approx \lambda_{2} \approx \lambda_{3}$ corresponds to an approximately isotropic tensor, with the ball component being dominant, and no main orientation present.

Consider two orientation tensors $A$ and $B$ and its summation $T=A+B$. After the decomposition of $T$ using (9), the component $\left(\lambda_{1}-\lambda_{2}\right) T_{1}$ is an estimate of the collinearity of the main eigenvectors of $A$ and $B$.

\section{Proposed Method}

The method proposed in this paper uses high frequency information extracted from wavelet analysis. For each scale $j$, we create a vector based on (5). This vector contains the high frequency value at vertical, horizontal and diagonal directions of the image $I$ at the position $p$ and scale $j$. Symmetric rank 2 tensors are then created as

$$
M_{j, p}=v_{j, p} v_{j, p}^{T} .
$$

We find the final tensor $M_{0, p}$ for each pixel of the original image using

$$
M_{0, p}=\sum_{j=1}^{n_{j}} k_{j} M_{j, p}
$$

to combine the tensors obtained at each scale $j$, where $n_{j}$ is the number of scales and $k_{j} \in \Re$ is the weight assigned to each scale, given by

$$
k_{j}=\frac{\sum_{n=1}^{n_{p}} \operatorname{Trace}\left(M_{j, n}\right)}{\sum_{k=1}^{n_{j}} \sum_{n=1}^{n_{p}} \operatorname{Trace}\left(M_{k, n}\right)},
$$

where $n_{p}$ is the number of pixels and Trace $\left(M_{j, p}\right)$ is the sum of the eigenvalues of $M_{j, p}$. The trace represents the amplification driven by the tensor to the unit sphere and is a good estimator of its importance. Thus, the tensor sum is weighted by the proportion of energy of each scale in the multiresolution pyramid.

In order to find $M_{j, p}$ in (11), we use bilinear interpolation of the tensor values, relative to each position $p$ in the initial image, at the subsampled image at scale $j$ to find the resulting tensor $M_{j, p}$ for each pixel of the initial image. This is 


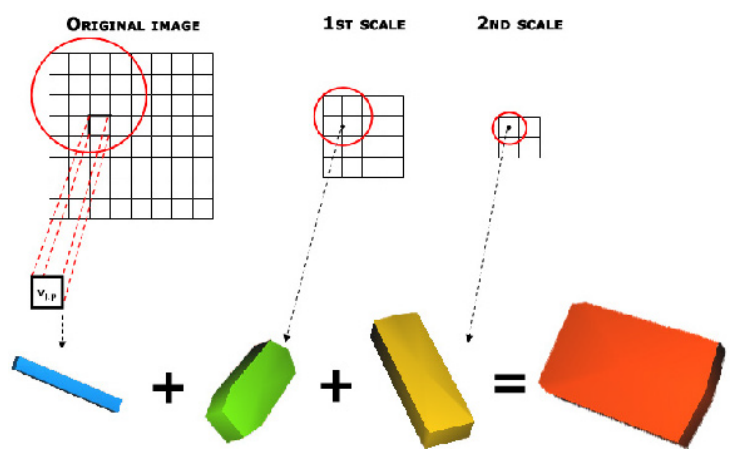

Fig. 1. A tensor is computed for each pixel in original image by a weighted sum of corresponding tensors in each scale. In this example, two wavelet decompositions are performed.

depicted in Fig. 1, where tensors are represented as superquadric glyphs whose longer axis shows the main direction.

Note that the tensor presented in (11) is a $3 \times 3$ positive symmetric matrix with real coefficients, and thus we may apply (9). We then find the main orientation component (spear) of the final orientation tensor for each pixel of the input image. This component indicates the collinearity of the interpolated tensors and provides interesting results.

\subsection{Implementation}

The proposed algorithm consists of three main steps: a discrete wavelet transform 810, a tensor field computation and a weighted sum of the computed tensors. The whole process is illustrated in Fig. 2 .

The number of scales to be used is a parameter of the algorithm. The DWT splits the image into three detail components and one scale component in the beginning of each iteration. In the next iteration, the same process is applied, using the resulting scale component as the input image.

For each pixel of the input image, its correspondent position at the current scale is computed with subpixel precision for each resolution. The four nearest pixels in a given resolution are used to compute the final tensor. The vectors $v_{j, p}$ described in (5) are computed for each of these pixels and then used to compute four spear type tensors. The final tensor for the subpixel position is obtained by combining these four tensors with bilinear interpolation. The pixel tensor is computed by combining the $n_{j}$ tensors as showed in (11).

The pixel tensors are decomposed and their eigenvalues are then extracted. The values $\lambda_{1}-\lambda_{2}$ are computed and normalized to form the output image. Color images are split into three monochromatic channels (Red, Green and Blue) and the proposed algorithm is applied to each channel separately. The tensors for each color channel are summed before eigen decomposition. 


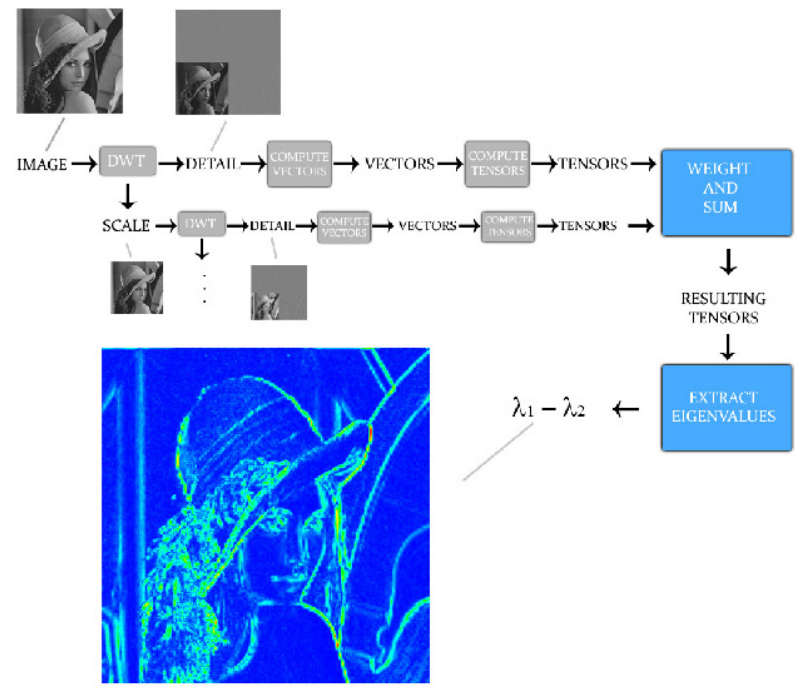

Fig. 2. Example of the proposed algorithm using Daubechies1 to decompose the image into two scales

The complexity of the whole process is $O\left(n_{j} \cdot n_{p}\right)$, where $n_{j}$ is the number of analyzed scales and $n_{p}$ the amount of input pixels. Thus, this is an efficient method that can be further parallelized.

\section{$5 \quad$ Experimental Results}

The first experiment consists of fixing an input image and varying the wavelet function and the amount of analyzed scales. This is shown in Figures 3 and 4 where the DWT is applied with different analyzing Daubechies filters and number of scales.

Comparing the Fig. 3b with the Fig. 3r, one may see that the number of scales is important to capture the coarse detail from the image. Note that the church's floor has low frequencies that cannot be detected using only one scale Fig. $3 \mathrm{~b}$. This is even clearer in Figures $4 \mathrm{~b}$ and 4 c. The ceiling is formed by coincident frequencies on its geometric details. These details can be better observed in Fig. 4r.

Changing the analyzing filter from Daubechies1 to Daubechies3 provides a better estimation of soft edge transitions. Figures $3 \mathrm{~b}, 3 \mathrm{~d}, 4 \mathrm{~b}$ and $4 \mathrm{~d}$ illustrate this behavior.

The resulting eigenvectors associated to the greatest eigenvalues $\lambda_{1}$ are shown in Fig. 5. The tensors eigenvectors are overlayed with the original image using the thermal color to indicate $\lambda_{1}-\lambda_{2}$. Note that they indicate regular patterns in high frequency regions.

In general, it can be noted that high frequencies occurring in the same region at different scales are highlighted by this method. The thermal coloring is a 


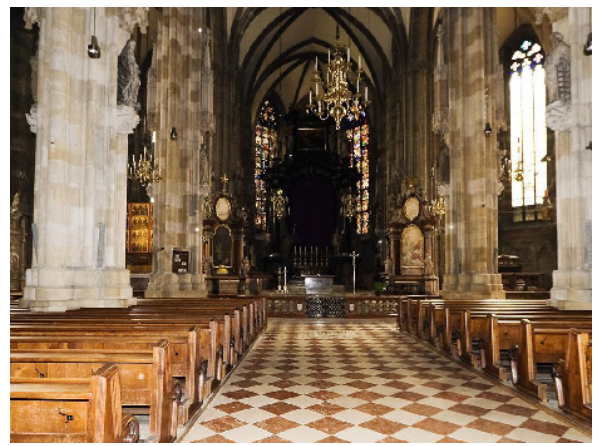

(a)

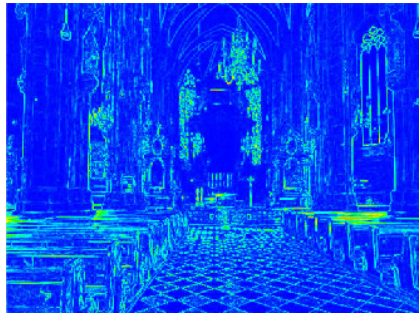

(b)

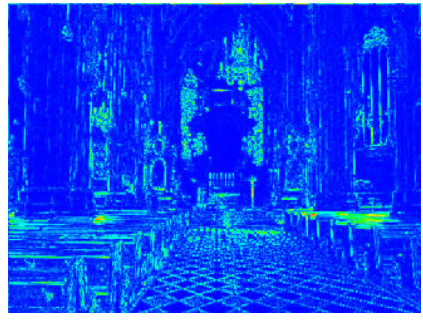

(d)

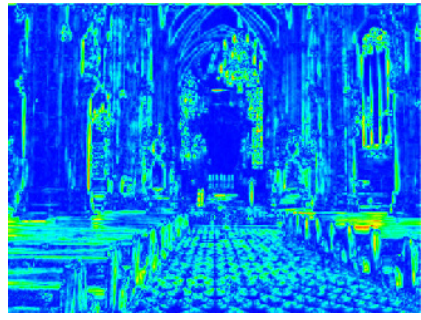

(c)

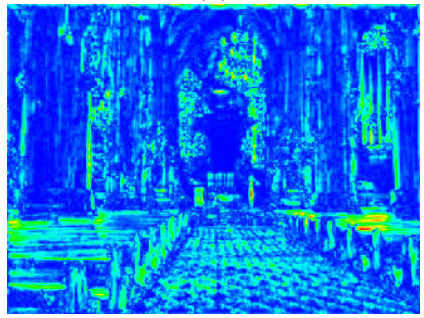

(e)

Fig. 3. (a) input image. (b) $\lambda_{1}-\lambda_{2}$ with Daubechies1 and 1 scale. c) Daubechies1 and 3 scales. d) Daubechies3 and 1 scale. e) Daubechies3 and 3 scales.

smooth transition from blue to red, where blue means absence of high frequencies, and red means presence of high frequencies. The green regions also indicate high frequencies, but not as intense as those indicated by red regions. The tensors obtained in the red regions have better estimation of higher frequencies.

The second experiment shows the time spent to apply the algorithm in color images. The Fig. [6] shows the time in seconds in function of the number of scales and image size. One may see the linear behavior of the algorithm, where the slope is the number of scales. However, it is important to note that the algorithm response time may be a bottleneck in real time applications if the number of pixels is high. All experiments were performed on an Intel Core2 Duo 1.8Ghz CPU using a 32 bit compiler. 


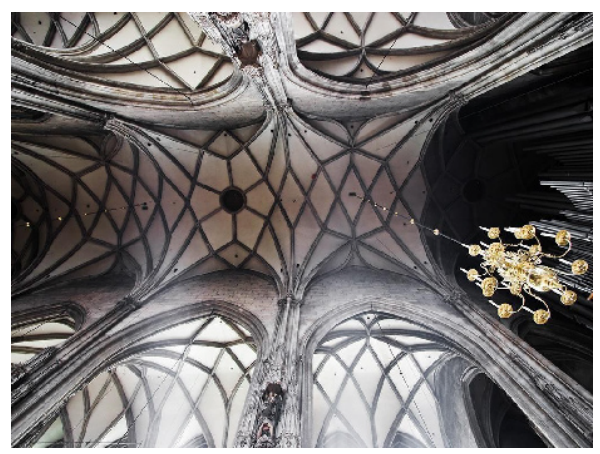

(a)

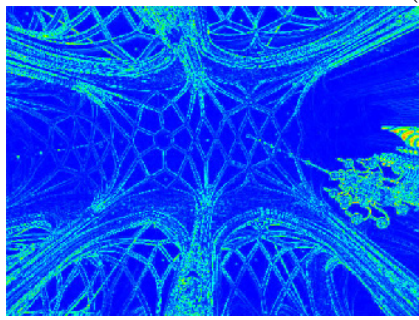

(b)

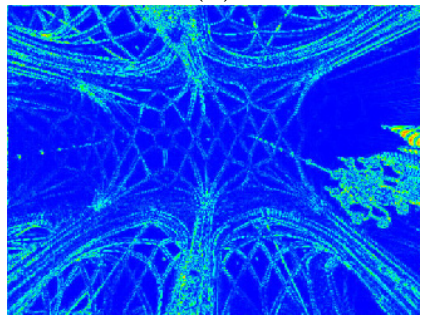

(d)

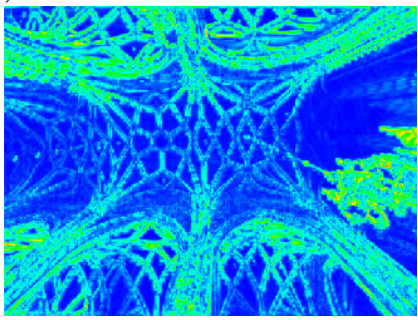

(c)

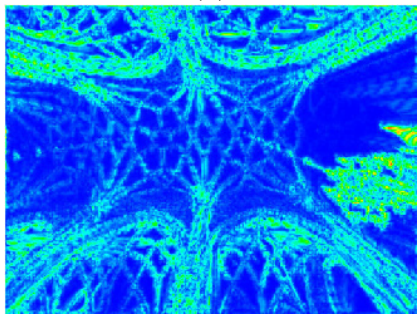

(e)

Fig. 4. (a) input image. (b) $\lambda_{1}-\lambda_{2}$ with Daubechies 1 and 1 scale. c) Daubechies 1 and 3 scales. d) Daubechies3 and 1 scale. e) Daubechies3 and 3 scales.
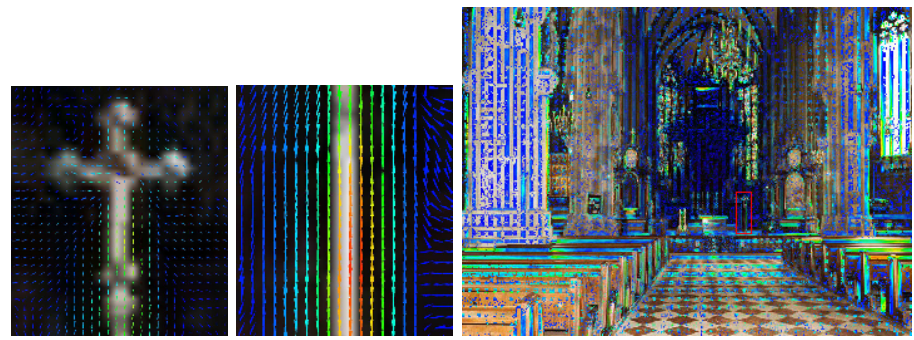

Fig. 5. Eigenvector field overlayed with the input image 
Pixels $\times$ Time

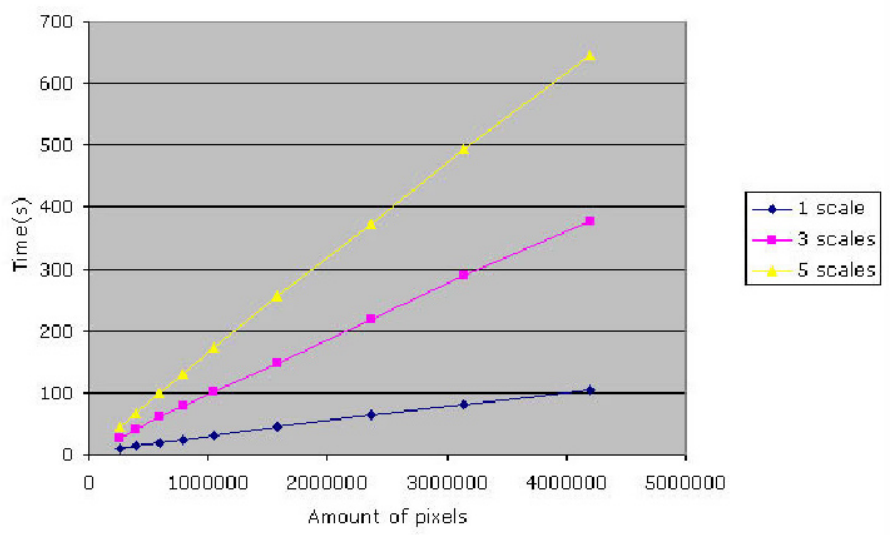

Fig. 6. Evaluation of the running time in function of the number of scales and amount of pixels of a color image

\section{Conclusions and Future Works}

A method for high frequency assessment and visualization was proposed. It is based on the DWT decomposition and detail information merging using orientation tensors. This multiresolution analysis showed to be suitable for detecting relevant edges and salient areas in an image. Due to the multivariate nature of tensors, the process can be easily applied in color images.

The experimental results show that the high frequency information can be inferred by varying the DWT filters and number of scales. Coincident frequencies in space domain are successfully highlighted. By tuning the number of scales, one may infer texture feature regions. As shown, the linear complexity is suitable for high performance processes.

The $\lambda_{1}-\lambda_{2}$ scalar field is one of the most used orientation alignment descriptors. However, other relations can be extracted from final pixel tensors. Future works should evaluate this remaining tensor information. As an example, there is promising information coded in the tensor eigenvectors. It is also interesting to investigate the tensor field instead of isolated tensors.

The discrete wavelet transform and the tensor summation can be easily parallelized. The use of rising technologies like GPGPUs and multicore CPUs turns this method attractive for high performance applications.

\section{Acknowledgments}

Authors thank Fundação de Amparo à Pesquisa do Estado de Minas Gerais /FAPEMIG and PROPESQ/UFJF for the financial support of this research. 


\section{References}

1. Knutsson, H.: Representing local structure using tensors. In: The 6th Scandinavian Conference on Image Analysis, Oulu, Finland, 244-251 Report LiTH-ISY-I-1019, Computer Vision Laboratory, Linköping University, Sweden (June 1989)

2. Fronthaler, H., Kollreider, K., Bigun, J.: Automatic image quality assessment with application in biometrics. In: CVPRW 2006: Proceedings of the 2006 Conference on Computer Vision and Pattern Recognition Workshop, Washington, DC, USA, p. 30. IEEE Computer Society Press, Los Alamitos (2006)

3. Wong, W.C.K., Chung, A.C.S.: Bayesian image segmentation using local isointensity structural orientation. IEEE Transactions on Image Processing 14(10), 1512-1523 (2005)

4. Han, Y., Shi, P.: An adaptive level-selecting wavelet transform for texture defect detection. Image Vision Comput. 25(8), 1239-1248 (2007)

5. Bigun, T.: Recognition by symmetry derivatives and the generalized structure tensor. IEEE Trans. Pattern Anal. Mach. Intell. 26(12), 1590-1605 (2004); Fellow-Josef Bigun and Student Member-Kenneth Nilsson

6. Schou, J., Dierking, W., Skriver, H.: Tensor based structure estimation in multichannel images. In: Geoscience and Remote Sensing Symposium, Proceedings. IGARSS, IEEE 2000 International, vol. 2, pp. 663-665 (2000)

7. van Vliet, L.J., Faas, F.G.A.: Multi-orientation analysis by decomposing the structure tensor and clustering. In: ICPR 2006: Proceedings of the 18th International Conference on Pattern Recognition, Washington, DC, USA, pp. 856-860. IEEE Computer Society Press, Los Alamitos (2006)

8. Mallat, S.: A Wavelet Tour of Signal Processing (Wavelet Analysis \& Its Applications), 2nd edn. Academic Press, London (1999)

9. Westin, C.F.: A Tensor Framework for Multidimensional Signal Processing. PhD thesis, Department of Electrical Engineering Linköping University (1994)

10. Barnard, H.J.: Image and Video Coding Using a Wavelet Decomposition. PhD thesis, Delft University of Technology, Department of Electrical Engineering, Information Theory Group, P.O.Box 5031, 2600 GA, Delft (1994) 